\title{
Direct observation of localized dipolar excitations on rough nanostructured surfaces
}

Bozhevolnyi, Sergey I.; Markel, V.A.; Coello, V.; KIm, W.; Shalaev, V.M.

Published in:

Physical Review B

Link to article, DOI:

10.1103/PhysRevB.58.11441

Publication date:

1998

Document Version

Publisher's PDF, also known as Version of record

Link back to DTU Orbit

Citation (APA):

Bozhevolnyi, S. I., Markel, V. A., Coello, V., KIm, W., \& Shalaev, V. M. (1998). Direct observation of localized dipolar excitations on rough nanostructured surfaces. Physical Review B, 58(17), 11441-11448.

https://doi.org/10.1103/PhysRevB.58.11441

\section{General rights}

Copyright and moral rights for the publications made accessible in the public portal are retained by the authors and/or other copyright owners and it is a condition of accessing publications that users recognise and abide by the legal requirements associated with these rights.

- Users may download and print one copy of any publication from the public portal for the purpose of private study or research.

- You may not further distribute the material or use it for any profit-making activity or commercial gain

- You may freely distribute the URL identifying the publication in the public portal 


\title{
Direct observation of localized dipolar excitations on rough nanostructured surfaces
}

\author{
Sergey I. Bozhevolnyi \\ Mikroelektronik Centret, Technical University of Denmark, Building 345 East, DK-2800 Lyngby, Denmark \\ Vadim A. Markel* \\ Department of Physics, New Mexico State University, Las Cruces, New Mexico 88003 \\ Victor Coello \\ Institute of Physics, Aalborg University, Pontoppidanstrde 103, DK-9220 Aalborg, Denmark
}

W. Kim and Vladimir M. Shalaev ${ }^{\dagger}$

Department of Physics, New Mexico State University, Las Cruces, New Mexico 88003

(Received 20 January 1998; revised manuscript received 17 April 1998)

\begin{abstract}
Using a photon scanning tunneling microscope (operating alternatively at the wavelengths of 594 and 633 $\mathrm{nm}$ ) with shear-force feedback we image the topography of silver colloid fractals simultaneously with a near-field intensity distribution. We observe that near-field optical images exhibit spatially localized (within $150-250 \mathrm{~nm}$ ) intensity enhancement by one to two orders of magnitude. These bright light spots are found to be sensitive to the light wavelength, polarization, and angle of incidence. We relate the observed phenomenon to the localization of resonant dipolar excitations in random nanostructured aggregates.
\end{abstract}

[S0163-1829(98)06941-0]

\section{INTRODUCTION}

Scattering of light by nanostructured surfaces results in a number of fascinating phenomena, among which one of the most interesting is the subwavelength light confinement. It is well known that electromagnetic fields are strongly enhanced (and, therefore, confined) near sharp corners and small scatterers, etc. ${ }^{1}$ Light confinement effects caused by subwavelength-sized surface defects have been studied theoretically $^{2,3}$ and observed with a near-field optical microscope. ${ }^{4}$ Specific surface structures (near-field holograms) can be used to further increase the effect of light confinement. ${ }^{5}$ In general, these phenomena are associated with the regime of the single scattering of light. In other words, an optical excitation near a certain subwavelength geometrical structure is independent of the surrounding large-scale geometry. Consequently, the optical enhancement is strongly influenced by the polarization of incident light and the local surface topography but is practically insensitive to the light wavelength and angle of incidence.

Scattering of light by rough nanostructured films and surfaces which are, typically, fractal is essentially different from the above phenomena. ${ }^{6-8}$ Resonant dipolar excitations in fractal structures also can be localized in subwavelengthsized regions but, being the result of multiple scattering, exhibit strong frequency and polarization dependence of their spatial location. ${ }^{9,10}$ This also means that the spatial location of light-induced dipole excitations is determined not only by the local topography, but also by the large-scale geometrical structure. Note that these features of light localization in fractals are similar to those observed for localization of surface plasmon polaritons (SPP's), ${ }^{11,12}$ which is also an interference phenomenon related to multiple scattering of SPP's (in the surface plane) caused by the surface roughness. ${ }^{13}$
The resonant optics of fractals has progressed immensely over the last decade, and many issues related to optical excitations in fractals have been theoretically clarified. ${ }^{6-10,14}$ It was established that dipolar eigenmodes which resonate at very close frequencies and, therefore, can be excited simultaneously by a monochromatic source (as long as they lie within a single resonance width) can have very different localization lengths, from the maximum size of a sample to the minimum roughness scale. ${ }^{9,15-18}$ This phenomenon has been referred to as inhomogeneous localization. ${ }^{15-17}$ However, in metal fractal nanocomposites the localization tends to increase, on average, when the wavelength changes from the plasmon resonance of an isolated metal spherule $(\sim 400 \mathrm{~nm})$ to the infrared, as was confirmed both theoretically ${ }^{9,19-21}$ and experimentally. ${ }^{22,23}$ Apart from the fact that, despite the chaotic behavior, the average localization length of dipolar eigenmodes tends to decrease with the wavelength, it should be noted that even if an eigenmode is delocalized (its gyration radius is of the order of the sample size), it can consist of several relatively small regions of high intensity which are spatially separated and, experimentally, viewed as "hot spots.",

Direct experimental evidence of the existence of localized dipolar excitations in fractal structures is still scarce. The use of near-field techniques is, probably, inevitable for direct imaging of these excitations, and interesting observations made with a photon scanning tunneling microscope (PSTM) have been reported for fractal metal colloid clusters ${ }^{22}$ and selfaffine surfaces. ${ }^{24}$ Optical modes of fractal aggregates were imaged in the constant-intensity mode (i.e., the detected optical signal was kept constant), and the observed spots were wavelength sized or even larger. ${ }^{22}$ Therefore, optical enhancement and subwavelength dimensions of these bright spots could be deduced only indirectly, from elevations of 
the probe over the sample surface. In addition, it is well known that PSTM images obtained in the constant-intensity mode can be rather misleading because of the strong influence of scattered waves propagating away from the sample ${ }^{25}$ and the nonmonotonic behavior of the total optical field. ${ }^{26}$ Note that alternative approaches, i.e., scanning in the constant plane or the constant distance modes, if chosen, should be also used very carefully: the first method is hardly suitable for deep surface profiles, ${ }^{26}$ and the second one can produce topographically induced artifacts. ${ }^{27,28}$ Finally, an uncoated fiber tip is a preferable choice for a PSTM probe, ${ }^{4,11,12}$ as opposed to a metal-coated tapered fiber, ${ }^{22}$ because it can be considered as a passive (nonperturbative) probe. $^{29}$

In this paper we report the results of the direct imaging of silver colloid aggregates deposited on a plane surface with a PSTM operating in the constant-distance mode for several different light wavelengths, polarizations, and angles of incidence. While the polarization and frequency sensitivity of dipolar excitations has been established with numerical simulations $^{14}$ and confirmed experimentally, ${ }^{22}$ its dependence on the angle of light incidence has not been reported in the literature. The idea to study this dependence was impelled by the similarity between dipolar excitations and localization of SPP's: both are associated with multiple scattering. The angular dependence of bright spots formed by scattered SPP's has been demonstrated and qualitatively related to the size of the surface area, within which SPP scattering contributes to the formation of a particular bright spot. $^{30}$

\section{SAMPLE PREPARATION}

Aggregates of silver colloid particles were prepared originally in solution by the borhydride method. ${ }^{31}$ Silver sol was generated by reducing silver nitrate with sodium borhydride. This reaction, given a proper concentration of reacting substances (see Ref. 31 for details), results in the appearance in the solution of many nearly spherical silver particles of approximately $7 \mathrm{~nm}$ in radius each. Initially, these particles are nonaggregated, and the extinction spectrum of this solution is peaked near the surface plasmon resonance of a spherical silver particle ( $\lambda \approx 400 \mathrm{~nm}$ in water); the color of nonaggregated colloid is opaque yellow. Addition of an adsorbent (fumaric acid) to the solution promotes aggregation of the small silver spherules into large fractal clusters built of many thousands of monomers. The kinetic of this aggregation process and the geometry of the resulting clusters are well studied. $^{32,33}$ It is known that the aggregation process is well described by the Meakin (cluster-cluster) aggregation model $^{34,35}$ in three dimensions. The clusters that form in the solution under normal conditions are fractal with fractal dimension $D \approx 1.8$.

After the process of aggregation was complete, the fractal aggregates were deposited onto a glass prism and the water was soaked out. The density of the aggregates in the solution was relatively low so that different clusters could be imaged individually. It should be noted that the process of the deposition of the samples on a surface and consequent evaporation of water results in a significant restructuring of clusters. Especially, the restructuring is important in the direction nor-

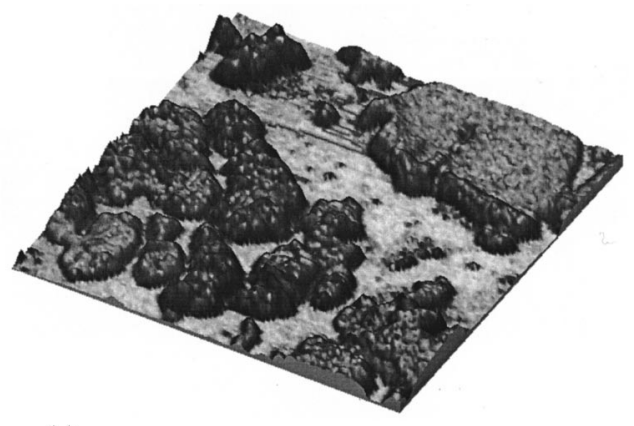

(a)

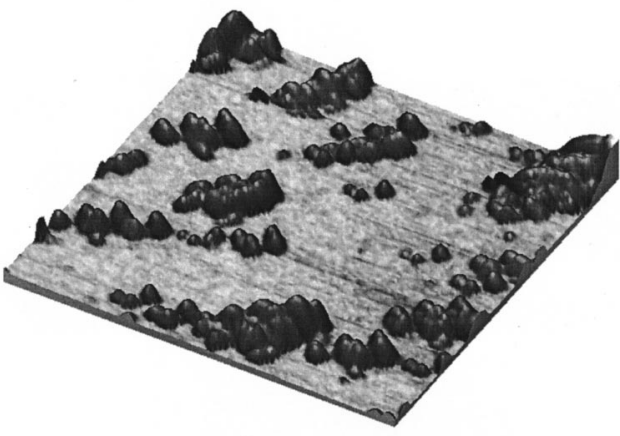

(b)

FIG. 1. (a) Typical topographical image (size $4 \times 4 \mu \mathrm{m}^{2}$, depth $127 \mathrm{~nm}$ ) of the sample surface showing clusters of silver particles and (b) zoom (size $1 \times 1 \mu \mathrm{m}^{2}$, depth $54 \mathrm{~nm}$ ) on a low-density area showing individual particles.

mal to the prism surface due to the collapse that can be caused by capillary forces and projection to two dimensions from three. However, as shown below, there are several indications that samples obtained as a result of this procedure retain the fractal geometry. More specifically, they possess the geometrical properties of self-affine surfaces.

The self-affine structures differ from self-similar fractal objects: to reveal scale invariance they require two different scaling factors, one in the surface plane and the other in the normal direction. The fractal dimension $D$ of a self-affine surface can be defined from the height-height correlation function

$$
g(R)=\left\langle[h(\mathbf{r}+\mathbf{R})-h(\mathbf{r})]^{2}\right\rangle \propto R^{2(3-D)},
$$

where $h(\mathbf{r})$ is the height of the surface at the point $\mathbf{r}$ $=(x, y)$, both $\mathbf{r}$ and $\mathbf{R}$ are vectors in the plane of the surface, and $2 \leqslant D \leqslant 3$.

Topographical images of our samples obtained with a commercial atomic-force microscope ${ }^{36}$ are shown in Fig. 1. Although in a smaller-resolution image [Fig. 1(a)] the holes and empty spaces characteristic for fractal structures are not resolved, comparison with the high-resolution image of a smaller area reveals the typical self-similar geometry. Note that the spatial resolution in Fig. 1(b) is less than $20 \mathrm{~nm}$ and individual particles are resolved. Another image of our sample obtained by the scanning electron microscopy is shown in Fig. 2. Again, the typical fractal geometry can be seen in this figure. 


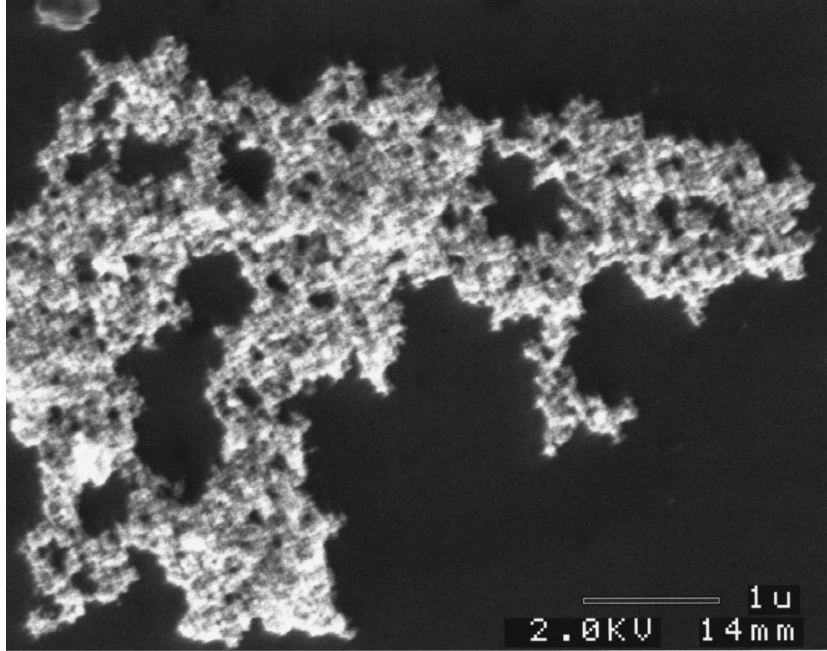

FIG. 2. A scanning electron micrograph of a sample prepared by the same method as the one shown in Fig. 1.

Although the topographical images shown in Figs. 1 and 2 reveal a visual resemblance of our samples to fractal structures, they cannot be analyzed quantitatively. In particular, the height-height correlation function (1) and the fractal dimension $D$ cannot be deduced from these images. To support the conjecture of the fractal geometry of our samples, we performed a numerical modeling of the aggregation and deposition processes. First, we have generated an ensemble of three-dimensional lattice cluster-cluster aggregates according to the Meakin algorithm; ${ }^{34}$ the details of our specific algorithm are described in Ref. 37 (in that paper, the same ensemble of fractal clusters as here was used). The fractal dimension of the generated clusters was close to 1.8. Then the clusters were deposited onto a plain surface and allowed to undergo a vertical collapse: the monomers that had an empty space directly beneath them were allowed to fall down until they hit the surface plane or another monomer. As a result, there were no empty spaces underneath monomers. Naturally, this projection from three to two dimensions resulted in a drastic restructuring of the clusters. An image of a single cluster-cluster aggregate with $N=10000$ monomers deposited on a surface is shown in Fig. 3. We calculated the height-height correlation function $g(R)$ for the deposited

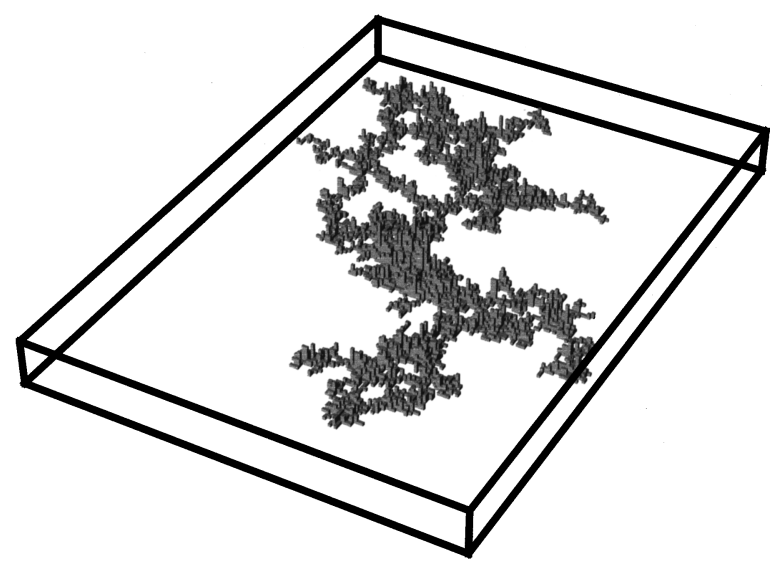

FIG. 3. A computer-generated cluster deposited on a surface (10 000 elementary blocks).

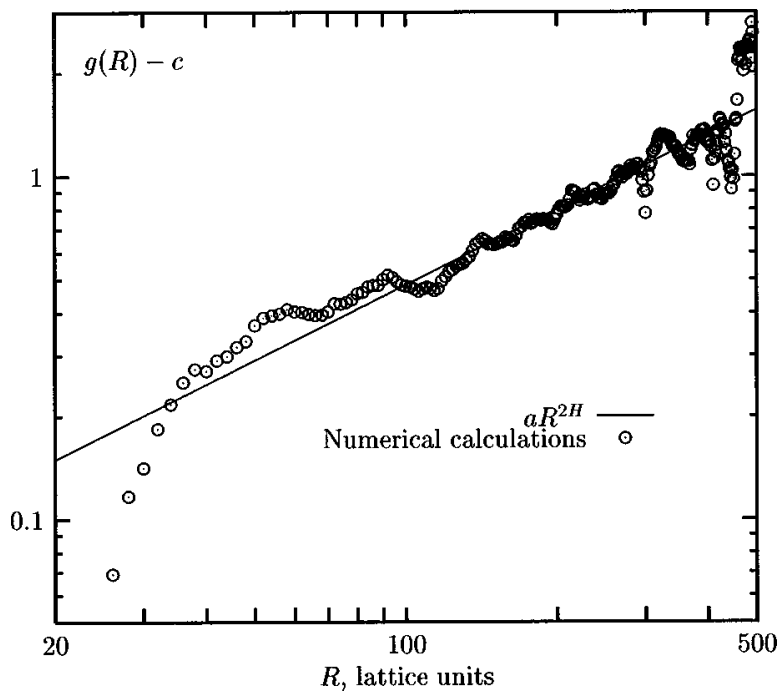

FIG. 4. The height-height correlation function (1) plotted vs the horizontal distance.

samples. It turned out that $g(R)$ has two characteristic correlation lengths. The first (short) length $l_{1}$ is, probably, associated with random noise; the second (large) length $l_{2}$ is the order of the overall sample size. When $R$ changes from 0 to $l_{1}$, the correlation function grows exponentially and saturates at some constant level $c$. For $l_{1}<R<l_{2}$, the function $[g(r)-c]$ grows according to the power law (1), as illustrated in Fig. 4. The scaling region extends from 30 to 400 lattice units and is well manifested. The corresponding fractal dimension of the samples, determined with the use of Eq. (1), is close to 2.6 .

\section{EXPERIMENT}

The experimental setup used in this work consists of a stand-alone PSTM combined with a shear-force-based feedback system, and is described in detail elsewhere. ${ }^{11,12}$ The polarization of the illuminating light beam that can be directed from any of the two He-Ne lasers $\left(\lambda_{1}=633 \mathrm{~nm}, P_{1}\right.$ $\left.=2 \mathrm{~mW} ; \lambda_{2}=594 \mathrm{~nm}, P_{2}=0.5 \mathrm{~mW}\right)$ is controlled with a $\lambda / 4$ plate and a linear polarizer. The $p$ - or $s$-polarized light beam (the electric field is parallel or perpendicular to the incidence plane, respectively) at one of the two wavelengths is used to illuminate a sample (placed on the base of a prism) under an angle that exceeds the angle of total internal reflection and can be varied. The incident beam is slightly focused onto the sample surface (focal length $\approx 500 \mathrm{~mm}$, spot size $\approx 400 \mu \mathrm{m})$ in order to increase the detected optical signal while providing reasonably uniform illumination of the scanning area $\left(4 \times 4 \mu \mathrm{m}^{2}\right)$. The near-field intensity distribution is probed with an etched fiber tip that ensures both efficient detection of the evanescent field components ${ }^{11,12}$ and relatively accurate imaging. ${ }^{38}$

The near-field optical images exhibited a spatially localized (within 150-250 nm) intensity enhancement of up to 10 times compared to the average background for both wavelengths and polarizations and for various angles of incidence (Figs. 5-8). The average optical signal was $\sim 50$ and $\sim 300 \mathrm{pW}$ for the yellow and red laser beams, respectively, and rapidly decreased with the tip-surface distance. The lat- 


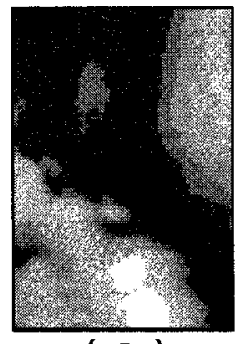

( a )

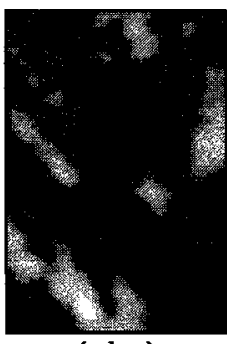

( b )

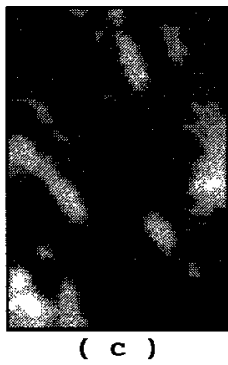

( c)
FIG. 5. Gray-scale topographical (a) and near-field optical (b),(c) images $\left(2 \times 3 \mu \mathrm{m}^{2}\right)$ taken with the shear-force feedback in the constant-distance mode (b) and just out of feedback in the constant-plane mode (c), when using the yellow $\left(\lambda_{2} \approx 594 \mathrm{~nm}\right) \mathrm{s}$ polarized laser beam incident on the sample surface under the angle of $48^{\circ}$. Depth of the topographical image is $120 \mathrm{~nm}$. Contrast, i.e., the relative difference between maximum and minimum detected optical signal of the optical images, is $98 \%$ (b) and $97 \%$ (c).

ter feature is inherent to the near-field imaging, and can, in principle, induce certain topographical artifacts in optical images obtained in the constant distance mode. ${ }^{27,28}$ We have carefully explored such a possibility by comparing optical images recorded in the constant-distance and the constantplane modes (Fig. 5). The only difference found between these images was related to the displacement of the fiber tip when getting out of contact with the sample surface [cf. Figs. 5(b) and 5(c)]. This means that the contrast observed in the near-field images is purely optical, i.e., not induced by topographical variations, ${ }^{27,28}$ a fact that can be explained by the rather strong and rapid variations of the near-field intensity in the surface plane. Once this fact is established, near-field imaging in the constant distance mode is preferable, since it allows one to achieve the best spatial resolution (which deteriorates with the increase of the tip-surface distance), and

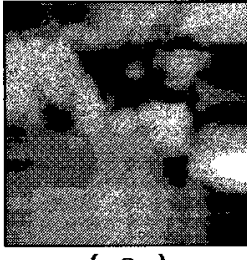

( a)

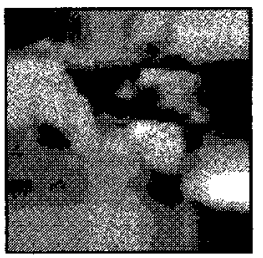

( d )
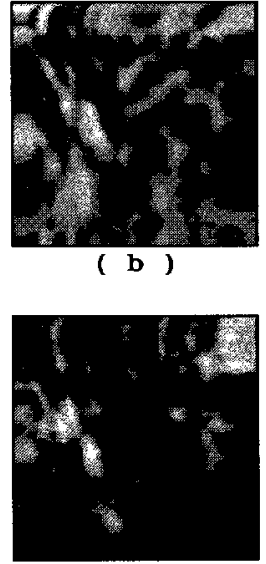

( e)
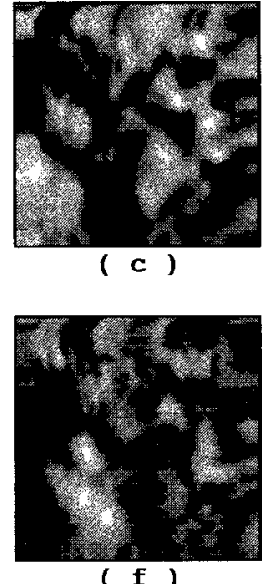

FIG. 6. Gray-scale topographical (a),(d) and near-field optical (b),(c),(e),(f) images $\left(4.4 \times 4.4 \mu \mathrm{m}^{2}\right)$ of the same area of the sample surface taken with the red $\left(\lambda_{1} \approx 633 \mathrm{~nm}\right) s$-polarized (b) and $p$-polarized (c) laser beams, and with the yellow $\left(\lambda_{1}\right.$ $\approx 594 \mathrm{~nm}$ ) $s$-polarized (e) and $p$-polarized (f) laser beams. The angle of beam incidence was $48^{\circ}$ for all images. The depth of the topographical images is $116 \mathrm{~nm}$. Contrast of the optical images is $87 \%$ (b), $86 \%$ (c), and $98 \%$ (e),(f). The topographical image (a) was taken simultaneously with the optical image (b); (d) was taken simultaneously with (e).
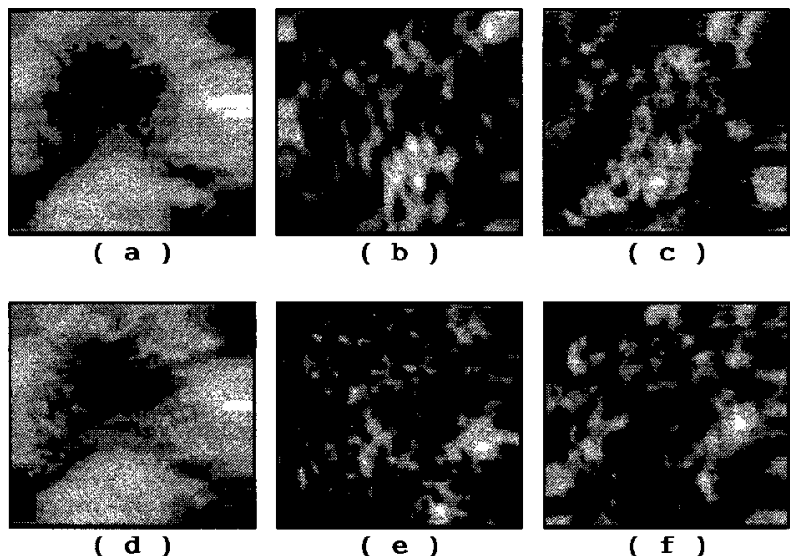

FIG. 7. Gray-scale topographical (a),(d) and near-field optical (b),(c),(e),(f) images $\left(4.4 \times 4.4 \mu \mathrm{m}^{2}\right)$ of the same area of the sample surface taken with red $s$-polarized (b) and $p$-polarized (c) laser beams, and with yellow $s$-polarized (e) and $p$-polarized (f) laser beams. The angle of beam incidence was $50^{\circ}$ for all images. The depth of the topographical images [(a) was taken simultaneously with (b) and (d) with (e)] is $85 \mathrm{~nm}$. Contrast of the optical images is $98 \%$ (b), $88 \%$ (c), $99 \%$ (e), and $97 \%$ (f).

to keep track of the imaged area when successive images of the same area are being taken and, thereby, to account for the eventual drift of the sample with respect to the fiber tip.

Actually, the stability of our setup appeared to be rather high, a circumstance that enabled us to obtain the near-field optical images for two wavelengths and two polarizations at the same surface area (Figs. 6 and 7). For all configurations and differently structured surface areas [cf. Figs. 6(a) and 7(a)], optical images exhibit a strong optical contrast with well-defined subwavelength-sized bright spots of round shape. The strong wavelength and polarization dependence of the spatial location of the bright spots is clearly seen in the obtained near-field images. These features are typical for interference phenomena in multiple scattering and consistent with the theoretical predictions of localized optical excitations in fractal structures. ${ }^{6-10}$ It should be also kept in mind that, due to the finite resolution of the PSTM with an uncoated fiber tip, ${ }^{39}$ localized optical excitations are expected to appear larger and less bright in the near-field images ${ }^{38}$ than they are in fact. Consequently, extended optical features, e.g., those related to the light scattering by clusters as a whole, are likely to become visible and to obscure, to some
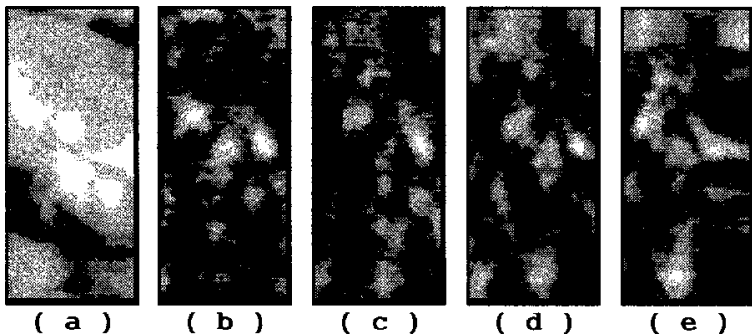

FIG. 8. Gray-scale topographical (a) and near-field optical (b),(c),(d),(e) images $\left(2 \times 4.4 \mu \mathrm{m}^{2}\right)$ of the same area of the sample surface taken with a red $s$-polarized laser beam incident on the sample surface under different angles: $48^{\circ}$ (b), $50^{\circ}$ (c), $52^{\circ}$ (d), and $54^{\circ}$ (e). The depth of the topographical image is $86 \mathrm{~nm}$. Contrast of the optical images is $95 \%$ (b), $93 \%$ (c), $98 \%$ (d), and $95 \%$ (e). 


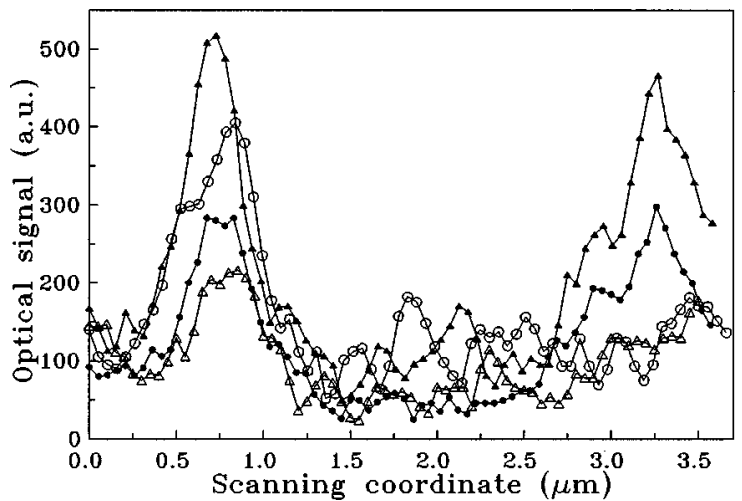

FIG. 9. Cross sections of optical images shown in Fig. 8 along the line connecting the left middle spot and the lower spot that are especially pronounced in Fig. 8(e). Open circles correspond to the angle of $48^{\circ}$, open triangles $50^{\circ}$, solid circles $52^{\circ}$, and solid triangles $54^{\circ}$.

extent, the structure of dipolar excitations (see optical images in Figs. 5-7).

Further, we investigated the dependence of the near-field optical images on the angle $\theta$ of beam incidence on the sample surface. Different surface areas have been scanned with the angle $\theta$ varying from $48^{\circ}$ to $58^{\circ}$ with a $2^{\circ}$ step. For both wavelengths and both polarizations, the tendency was that the bright spots changed their contrast with $\theta$ (Fig. 8). However, contrary to what has been observed for localization of SPP's, ${ }^{30}$ different bright spots showed different angular dependences. For example, the lowest spot in Fig. 8(e) is absent in Fig. 8(b), and the left middle spot first decreased and then increased again its brightness with the increase of $\theta$, whereas the right middle spot is hardly changed [Figs. $8(\mathrm{~b})-8(\mathrm{e})]$. The angular variations in the intensity distributions of the first two spots are shown in Fig. 9. We believe that the difference in the angular dependences observed for different bright spots is related to the difference in the coherent lengths of the appropriate dipolar eigenmodes.

\section{RESULTS AND DISCUSSION}

The observed subwavelength localization of the electromagnetic field and its enhancement are anticipated experimental results. We have directly imaged localization areas ("bright spots") of the size as small as $150 \mathrm{~nm}$. It should be noted that the actual size of these bright spots might be even smaller because of the finite resolution of the PSTM. The observed spectral and polarization dependence of the spatial positions of these bright spots has also been expected. However, the dependence of the positions of the bright spots on the angle of incidence is, as far as we know, a new phenomenon and has not been observed or discussed theoretically in the literature. The origin of this effect is also not as clear as that of the spectral and polarization dependence. It is evident that changing the angle of incidence for the $s$-polarized light changes only the phase velocity of the evanescent wave in the plane of the surface but keeps both polarization and frequency of this wave constant.

To gain some understanding of this phenomenon we refer to the theory built in Refs. 9 and 14 which is based on a simple dipole approximation and perform some numerical calculations with the use of this approximation. Let us first briefly review the dipole approximation. We model an arbitrary cluster as a set of point dipoles (monomers) located in points $\mathbf{r}_{i}(i=1,2, \ldots, N)$ that can interact with the incident (evanescent) field and with each other. Each dipole has a scalar polarizability $\alpha$ which, in the first approximation, is given by the Lorenz-Lorentz formula $\alpha=R_{m}^{3}(\epsilon-1) /(\epsilon$ $+2)$, where $\epsilon$ is the dielectric constant of silver taken at the frequency of the laser beam, $\omega$, and $R_{m}$ is a constant of the dimensionality of length. If we model a sample of the total volume $V$ with $N$ point dipoles, we can find $R_{m}$ from the conservation of volume: $V=N(4 \pi / 3) R_{m}^{3}$. Each monomer is characterized by a linear dipole moment $\mathbf{d}_{i}$ which oscillates at the frequency $\omega$. The dipole moments are coupled to each other and to the evanescent field by the coupled-dipole equation $^{40,41}$

$$
\mathbf{d}_{i}=\alpha\left[\mathbf{E}_{\mathrm{ev}}\left(\mathbf{r}_{i}\right)+\sum_{j \neq i} \hat{G}\left(\mathbf{r}_{i}-\mathbf{r}_{j}\right) \circ \mathbf{d}_{j}\right],
$$

where $\hat{G}\left(\mathbf{r}_{i}-\mathbf{r}_{j}\right) \circ \mathbf{d}_{j}$ is the dipole radiation field created by the $j$ th dipole in point $\mathbf{r}_{i}$, including the near-, intermediate-, and far-zone terms (see, for example, Ref. 41) and $\mathbf{E}_{\mathrm{ev}}\left(\mathbf{r}_{i}\right)$ is the incident (evanescent) field taken at the point $\mathbf{r}_{i} . \hat{G}(\mathbf{r})$ is the dyadic Green's function for the vector wave equation. ${ }^{41}$ The system of linear equations (2) can be solved to find all the dipole moments. The electric field in an arbitrary point of observation $\mathbf{R}$ can be calculated according to

$$
\mathbf{E}(\mathbf{R})=\sum_{i=1}^{N} \hat{G}\left(\mathbf{R}-\mathbf{r}_{i}\right) \circ \mathbf{d}_{i}
$$

The experimentally measurable value, the local intensity $I(\mathbf{R})$, can be calculated according to $I(\mathbf{R})=|\mathbf{E}(\mathbf{R})|^{2}$.

To investigate the influence of the incidence angle on the local intensity, $I(\mathbf{R})$, it is useful to consider the eigenvector expansion of the solution to Eq. (2). As was shown in Ref. 9, this expansion has the form

$$
\mathbf{d}_{i}=\sum_{n=1}^{3 N} \frac{\mathbf{x}_{i}^{(n)}\left[\sum_{j=1}^{N} \mathbf{x}_{j}^{(n) *} \cdot \mathbf{E}_{\mathrm{ev}}\left(\mathbf{r}_{j}\right)\right]}{\left[\sum_{j=1}^{N}\left(\mathbf{x}_{j}^{(n)}\right)^{2}\right]\left[1 / \alpha(\omega)-w_{n}\right]}
$$

where $\mathbf{x}_{i}^{(n)}$ are the (possibly complex) components of the $n$th eigenvector of Eq. (2) and $w_{n}$ are the corresponding eigenvalues. The generalized resonance condition can be written as $\operatorname{Re}\left[1 / \alpha(\omega)-w_{n}\right] \leqslant \gamma_{n}$ where $\gamma_{n}$ is the width of the corresponding resonance. The $n$th resonance frequency can be found by solving the equation $\operatorname{Re}\left[1 / \alpha\left(\omega_{n}\right)-w_{n}\right]=0$ and the corresponding width, according to $\gamma_{n}=\operatorname{Im}\left[1 / \alpha\left(\omega_{n}\right)-w_{n}\right]$. The expansion for $I(\mathbf{R})$ has the form

$I(\mathbf{R})$

$$
=\sum_{n, m=1}^{3 N} F_{m n}(\mathbf{R}) \frac{\left[\sum_{i=1}^{N} \mathbf{x}_{i}^{(n) *} \cdot \mathbf{E}_{\mathrm{ev}}\left(\mathbf{r}_{i}\right)\right]\left[\sum_{i=1}^{N} \mathbf{x}_{i}^{(n)} \cdot \mathbf{E}_{\mathrm{ev}}^{*}\left(\mathbf{r}_{i}\right)\right]}{\left[1 / \alpha(\omega)-w_{n}\right]\left[1 / \alpha(\omega)-w_{m}\right]^{*}}
$$

$$
F_{m n}(\mathbf{R})=\frac{\left[\sum_{i=1}^{N} \hat{G}\left(\mathbf{R}-\mathbf{r}_{i}\right)^{\circ} \mathbf{x}_{i}^{(n)}\right] \cdot\left[\sum_{i=1}^{N} \hat{G}\left(\mathbf{R}-\mathbf{r}_{i}\right)^{\circ} \mathbf{x}_{i}^{(m)}\right]^{*}}{\left[\sum_{i=1}^{N}\left(\mathbf{x}_{i}^{(n)}\right)^{2}\right]\left[\sum_{i=1}^{N}\left(\mathbf{x}_{i}^{(m)}\right)^{2}\right]^{*}} .
$$


(a) $\theta=48^{\circ}$

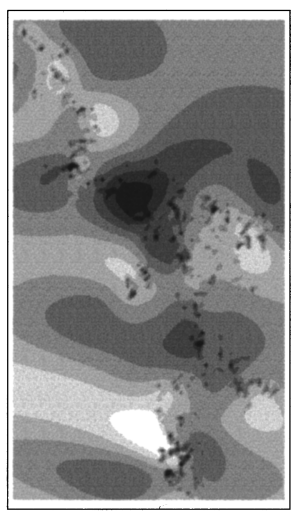

(b) $\theta=50^{\circ}$

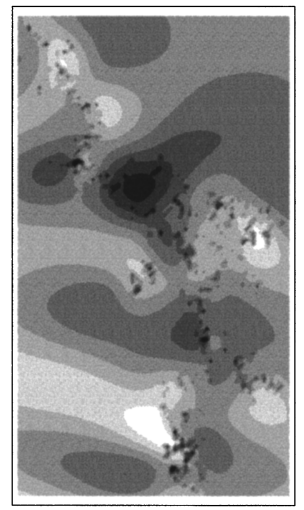

(c) $\theta=52^{\circ}$

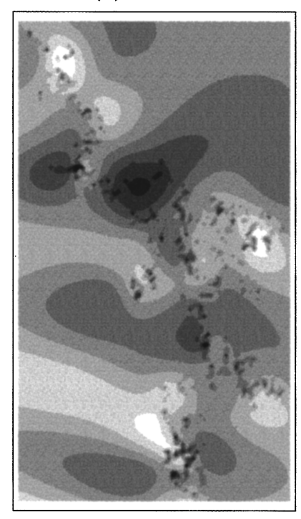

(d) $\theta=54^{\circ}$

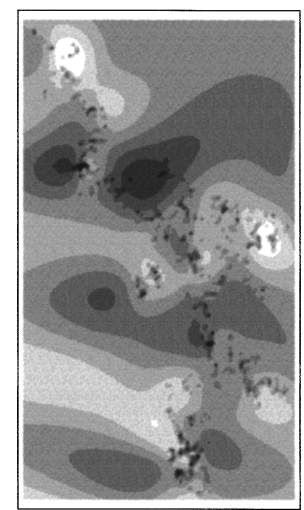

FIG. 10. Computer-generated optical image of a cluster taken in the constant-distance mode for $s$-polarized light with $\lambda=633 \mathrm{~nm}$ at different angles of the laser beam incidence. The size of the sample is $0.65 \times 1.17 \mu \mathrm{m}^{2}$; the distance between the tip and surface is $100 \mathrm{~nm}$. The topography of the cluster can be seen as a set of little black points.

The only term in Eqs. (4) and (5) that depends on the angle of incidence of the laser beam is $\mathbf{E}_{\mathrm{ev}}(\mathbf{r})$ :

$$
\mathbf{E}_{\mathrm{ev}}(\mathbf{r})=\mathbf{E}_{0} \exp \left[\frac{\omega}{c}\left(-z \sqrt{n^{2} \sin ^{2} \theta-1}+i x n \cos \theta\right)\right]
$$

where $\theta$ is the angle of incidence (with respect to a perpendicular to the prism surface), the $z$ axis is normal to the prism surface, and the $x$ axis coincides with the longitudinal direction of the evanescent field propagation. The complete internal reflection condition is $n \sin \theta>1$. Evidently, a change of the incidence angle affects both the normal and the longitudinal components of the evanescent wave vector. However, in our experiments the vertical depth of samples $(\sim 130 \mathrm{~nm})$ was significantly smaller than the characteristic distance of the vertical decay of the evanescent field $l_{z}$ (for example, for $\lambda=633 \mathrm{~nm}$ and $\theta=48^{\circ}, \quad l_{z} \approx 1200 \mathrm{~nm}$ ). Therefore, both bottom and top layers of a cluster are excited by a field of almost constant intensity. ${ }^{42}$

The diagonal terms in Eq. (5) $(m=n)$ represent the field patterns associated with single modes while the off-diagonal terms $(m \neq n)$ are due to intermode interference. It is evident that in the single-mode regime (when only one mode, say, $n=M$, is in resonance and can be effectively excited) varying $\theta$ can result only in changing the overall brightness of the optical image but not in redistribution of the local intensity. Mathematically, it follows from the fact that the local field distribution becomes proportional to $F_{M M}(\mathbf{R})$ in this case, which does not depend on $\theta$. However, if several modes can be excited simultaneously, the optical image is governed by several functions $F_{m n}(\mathbf{R})$, each with its own weight, which depends on $\theta$. Therefore, varying $\theta$ can result in a redistribution of the local intensity (for example, by increasing the intensity due to a certain mode and decreasing the input of other modes). The experimentally observed angular dependence indicates that several dipole modes were excited simultaneously in our samples. It also carries certain information about the localization of the eigenmodes. Indeed, if the eigenmodes were homogeneous and delocalized over the whole sample (more precisely, if $\left|\mathbf{x}_{i}^{(n)}\right|$ do not depend on $i$ ), the redistribution of the intensity between modes would not occur, as can be seen from the approximate evaluation of the coefficients in the numerator of Eq. (5). On the other hand, the modes that are localized in very small areas with characteristic sizes $L \ll \lambda / 2 \pi n \cos \theta$ are also not sensitive to $\theta$. From the mathematical form of the coefficients in Eq. (5) and the phase dependence of $\mathbf{E}_{\mathrm{ev}}$ one can conclude that the modes that have several isolated zones of high local intensity separated by distances $\geqslant \lambda / 2 \pi n \cos \theta$ are most sensitive to the change of $\theta$. In Figs. 8(b)-8(e) one can see several such "hot zones" that belong to different dipolar eigenmodes, as well as the redistribution of the local intensity with varying $\theta$. The characteristic distance between the bright spots in Fig. 8(b) is approximately $500 \mathrm{~nm}$, while $\lambda / 2 \pi n \cos \theta$ $\approx 100 \mathrm{~nm}$.

We also performed a numerical modeling of the dependence of the local intensity distribution on the angle of incidence, $\theta$. The process of aggregation and deposition of a cluster on the surface was modeled on a computer as described in Sec. II. Then we solved the coupled dipole equation (2) and calculated the local intensity $I(\mathbf{R})$ according to Eq. (5) in the constant-distance mode. The vertical distance between the point of observation, $\mathbf{R}$, and the highest point of the surface right beneath the point of observation was kept at a constant value $h=100 \mathrm{~nm}$, which is comparable to the typical optical aperture of experimental PSTM's. The wavelength of the incident radiation was taken to be equal to the red laser line $(633 \mathrm{~nm})$. The tip was supposed to be pointlike and nonperturbing in this calculation. The result is shown in Fig. 10. The angular dependence similar to the one observed in the experiment is clearly visible in these images. The characteristic distance between the three bright spots that change their intensity with $\theta$ (one in the left upper corner, the other in the middle of the bottom part, and the third in the center of the right part of the image) is approximately $600 \mathrm{~nm}$ which is substantially larger than $\lambda / 2 \pi n \cos \theta$. The maximum local intensity enhancement factor (calculated over ten different samples, only one of which is shown in Fig. 10) is $\approx 40$ in this calculation. The contrast of optical images is more than $50 \%$. It should be noted that the maximum enhancement factor, as well as the optical contrast, rapidly increases when $h$ decreases (the corresponding numerical data are not shown).

It is rather cumbersome to relate the experimentally observed enhancement (of up to 10 times) in the near-field 
optical images to the calculated local intensity enhancement (in the absence of a fiber tip). The experimental value corresponds to the ratio between the maximum signal and the average background. If one defines the enhancement with respect to the incident evanescent field, then the background value should be taken at areas without clusters (as long as this can be judged from the topographical images). This would increase the observed enhancement up to 20 times. Next, it is important (but very difficult) to take into account the fact that the spatial resolution of the near-field microscope is limited. In general, one has to determine the transfer function of the fiber tip used (assuming that it can be considered as a passive probe ${ }^{29}$ ), which is a challenging problem itself. $^{43}$ Taking into account that, for the spatial period of $\sim 300 \mathrm{~nm}$ of the intensity interference pattern, the contrast correction factor is $\sim 2,{ }^{43}$ one can deduce that the enhancement corresponding to the experimental images is at least 40 times. Alternatively, one can consider the imaging of bright spots with uncoated fiber tips that has been modeled theoretically. ${ }^{38}$ In these calculations, there was found a good correspondence (in the shape and value of half-widths of distributions) between the field intensity distribution at 100 $\mathrm{nm}$ and the signal dependence measured at $5 \mathrm{~nm}$ distance. Comparing our calculations for $h=100 \mathrm{~nm}$ and the experimental results one finds also similar values of enhancement and sizes of bright spots (cf. Figs. 8 and 10). Overall, there is a qualitative and even quantitative (though not straightforward) agreement between our experimental results and numerical simulations.

\section{SUMMARY}

In summary, we have presented the near-field optical images of optical excitations in silver colloid fractals for two wavelengths, $s$ and $p$ polarizations, and different angles of illuminating beam incidence. These images exhibited subwavelength-sized bright spots whose positions were found to be sensitive to the light wavelength, polarization, and angle of incidence. We have related the observations to the localization of resonant dipolar excitations in fractal aggregates. A feature of this phenomenon, viz., the angular dependence of the strength of localized optical excitations, has been discussed. Preliminary numerical simulations have been carried out and the intensity distribution calculated for different angles of incidence has been found to bear a close resemblance to the experimental near-field images. This feature should be definitely investigated further as it might be important for practical applications associated, for example, with persistent holes induced by laser radiation in the spectra of fractals. ${ }^{23,44}$

\section{ACKNOWLEDGMENTS}

This work was supported in part by NSF Grant Nos. DMR-9623663 and DMR-0500258 and by NATO Grant No. CRG-950097. Computational facilities were provided by the National Center for Supercomputing Applications under Grant No. PHY980006N. Acknowledgment is also made to the donors of the Petroleum Research Fund, administered by the ACS, for partial support of this research.
*Also with the Institute of Automation and Electrometry, Siberian Branch of RAS, 630090 Novosibirsk, Russia.

† Author to whom correspondence should be addressed. Electronic address: vshalaev@nmsu.edu.

${ }^{1}$ J. D. Jackson, Classical Electrodynamics (Wiley, New York, 1975).

${ }^{2}$ C. Girard, A. Dereux, O. J. F. Martin, and M. Devel, Phys. Rev. B 52, 2889 (1995).

${ }^{3}$ O. J. F. Martin, C. Girard, and A. Dereux, Phys. Rev. Lett. 74, 526 (1995).

${ }^{4}$ J.-C. Weeber, E. Bourillot, A. Dereux, J.-P. Goudonnet, Y. Chen, and C. Girard, Phys. Rev. Lett. 77, 5332 (1996).

${ }^{5}$ S. I. Bozhevolnyi and B. Vohnsen, Phys. Rev. Lett. 77, 3351 (1996).

${ }^{6}$ S. Alexander and R. Orbach, J. Phys. (France) Lett. 43, L625 (1982).

${ }^{7}$ V. A. Markel, L. S. Muratov, M. I. Stockman, and T. F. George, Phys. Rev. B 43, 8183 (1991).

${ }^{8}$ M. I. Stockman, T. F. George, and V. M. Shalaev, Phys. Rev. B 44, 115 (1991)

${ }^{9}$ V. A. Markel, V. M. Shalaev, E. B. Stechel, W. Kim, and R. L. Armstrong, Phys. Rev. B 53, 2425 (1996).

${ }^{10}$ V. M. Shalaev, E. Y. Poliakov, and V. A. Markel, Phys. Rev. B 53, 2437 (1996).

${ }^{11}$ S. I. Bozhevolnyi, I. I. Smolyaninov, and A. V. Zayats, Phys. Rev. B 51, 17916 (1995).

${ }^{12}$ S. I. Bozhevolnyi, Phys. Rev. B 54, 8177 (1996).

${ }^{13}$ K. Arya, Z. B. Su, and J. L. Birman, Phys. Rev. Lett. 54, 1559 (1985).

${ }^{14}$ V. M. Shalaev, Phys. Rep. 272, 61 (1996).
${ }^{15}$ M. I. Stockman, L. N. Pandey, and T. F. George, Phys. Rev. B 53, 2183 (1996).

${ }^{16}$ M. I. Stockman, Phys. Rev. E 56, 6494 (1997).

${ }^{17}$ M. I. Stockman, Phys. Rev. Lett. 79, 4562 (1997).

${ }^{18}$ The localization of eigenmodes has been studied theoretically in the quasistatic approximation. Thus, the effects of retardation on the localization have not been clarified to the best of our knowledge.

${ }^{19}$ E. Y. Poliakov, V. M. Shalaev, V. A. Markel, and R. Botet, Opt. Lett. 21, 1628 (1996).

${ }^{20}$ V. M. Shalaev, E. Y. Poliakov, V. A. Markel, and R. Botet, Physica A 241, 249 (1997).

${ }^{21}$ E. Y. Poliakov, V. A. Markel, V. M. Shalaev, and R. Botet, Phys. Rev. B 57, 14901 (1998).

${ }^{22}$ D. P. Tsai, J. Kovacs, Z. Wang, M. Moskovits, V. M. Shalaev, J. S. Suh, and R. Botet, Phys. Rev. Lett. 72, 4149 (1994); V. M. Shalaev and M. Moskovits, ibid. 75, 2451 (1995).

${ }^{23}$ V. P. Safonov, V. M. Shalaev, V. Markel, Y. E. Danilova, N. N. Lepeshkin, W. Kim, S. G. Rautian, and R. L. Armstrong, Phys. Rev. Lett. 80, 1102 (1988).

${ }^{24}$ M. Moskovits recently informed one of the authors (V.S.) about his PSTM (with shear-force feedback) studies of optical excitations on a silver self-affine surface.

${ }^{25}$ P. M. Adam, L. Salomon, F. de Fornel, and J. P. Goudonnet, Phys. Rev. B 48, 2680 (1993).

${ }^{26}$ C. Girard and D. Courjon, Surf. Sci. 382, 9 (1997).

${ }^{27}$ B. Hecht, H. Bielefeldt, Y. Inouye, D. W. Pohl, and L. Novotny, J. Appl. Phys. 81, 2492 (1997).

${ }^{28}$ S. I. Bozhevolnyi, J. Opt. Soc. Am. B 14, 2254 (1997). 
${ }^{29}$ J. C. Weeber, F. de Fornel, and J. P. Goudonnet, Opt. Commun. 126, 285 (1996).

${ }^{30}$ S. I. Bozhevolnyi, B. Vohnsen, I. I. Smolyaninov, and A. V. Zayats, Opt. Commun. 117, 417 (1995).

${ }^{31}$ J. A. Creighton, C. G. Blatchford, and M. G. Albreht, J. Chem. Soc., Faraday Trans. 2 75, 790 (1979).

${ }^{32}$ D. A. Weitz and M. Oliveria, Phys. Rev. Lett. 52, 1433 (1984).

${ }^{33}$ R. Jullien, M. Kolb, and R. Botet, J. Phys. (France) Lett. 45, L211 (1984).

${ }^{34}$ P. Meakin, Phys. Rev. Lett. 51, 1119 (1983).

${ }^{35}$ P. Meakin and F. Family, Phys. Rev. A 36, 5498 (1987).

${ }^{36}$ DME dualscope, Danish Micro Engineering A/S, Herlev, Denmark.

${ }^{37}$ V. A. Markel, V. M. Shalaev, E. Y. Poliakov, and T. F. George, Phys. Rev. E 55, 7313 (1997).

${ }^{38}$ S. I. Bozhevolnyi, B. Vohnsen, E. A. Bozhevolnaya, and S. Bern- tsen, J. Opt. Soc. Am. A 13, 2381 (1996).

${ }^{39}$ S. I. Bozhevolnyi and B. Vohnsen, J. Opt. Soc. Am. B 14, 1656 (1997).

${ }^{40}$ E. M. Purcell and C. R. Pennypacker, Astrophys. J. 186, 705 (1973).

${ }^{41}$ B. T. Draine, Astrophys. J. 333, 848 (1988).

${ }^{42}$ If $l_{z}$ is much smaller than the vertical depth of a sample (e.g., at very large incidence angles), only bottom layers of the sample that are, typically, more homogeneous would be excited by the incident waves. The higher layers are excited only by secondary (scattered) fields, in this case.

${ }^{43}$ V. Coello, S. I. Bozhevolnyi, and F. A. Pudonin, Proc. SPIE 3098, 536 (1997).

${ }^{44}$ A. V. Butenko, P. A. Chubakov, Y. E. Danilova, S. V. Karpov, A. K. Popov, S. G. Rautian, V. P. Safonov, V. V. Slabko, V. M. Shalaev, and M. I. Stockman, Z. Phys. D 17, 283 (1990). 